\title{
Video Endoscopic Inguinal Lymphadenectomy (VEIL): Minimally Invasive Resection of Inguinal Lymph Nodes
}

\author{
M. Tobias-Machado, Alessandro Tavares, Wilson R. Molina Jr, Pedro H. Forseto Jr, Roberto \\ V. Juliano, Eric R. Wroclawski
}

Section of Urology, ABC Medical School, Santo Andre, Sao Paulo, Brazil

\begin{abstract}
Objectives: Describe and illustrate a new minimally invasive approach for the radical resection of inguinal lymph nodes. Surgical Technique: From the experience acquired in 7 operated cases, the video endoscopic inguinal lymphadenectomy (VEIL) technique was standardized in the following surgical steps: 1) Positioning of the inferior member extended in abduction, 2) Introduction of 3 work ports distal to the femoral triangle, 3) Expansion of the working space with gas, 4) Retrograde separation of the skin flap with a harmonic scalpel, 5) Identification and dissection of the long saphenous vein until the oval fossa, 6) Identification of the femoral artery, 7) Distal ligature of the lymph node block at the femoral triangle vertex, 8) Liberation of the lymph node tissue up to the great vessels above the femoral floor, 9) Distal ligature of the long saphenous vein, 10) Control of the saphenofemoral junction, 11) Final liberation of the surgical specimen and endoscopic view showing that all the tissue of the region was resected, 12) Removal of the surgical specimen through the initial orifice, 13) Vacuum drainage and synthesis of the incisions.

Comments: The VEIL technique is feasible and allows the radical removal of inguinal lymph nodes in the same limits of conventional surgery dissection. The main anatomic repairs of open surgery can be identified by the endoscopic view, confirming the complete removal of the lymphatic tissue within the pre-established limits. Preliminary results suggest that this technique can potentially reduce surgical morbidity. Oncologic follow-up is yet premature to demonstrate equivalence on the oncologic point of view.
\end{abstract}

Key words: penile cancer; groin; lymphadenectomy; video-assisted surgery

Int Braz J Urol. 2006; 32: 316-21

\section{INTRODUCTION}

Inguinal lymphadenectomy is indicated in patients presenting penile and urethral cancer, after local treatment, when there is a lymph node mass that does not disappear with antibiotic therapy, or when palpable lymph nodes appear in the postoperative follow-up or when there are risk factors for the development of inguinal metastasis (prophylactic lymphadenectomy). This operation is frequently performed through a bilateral inguinal incision from the iliac crest until the pubic tubercle. There is, however, a high morbidity regarding the dissected skin flap to access the inguinal lymph nodes, as well as skin necrosis and local infection, and depending on the extension of the lymphadenectomy, higher frequency of edema in inferior members, lymphocele, lymphedema and lymphorea (1).

Trying to reduce the morbidity of this radical operation the literature shows surgical alternatives 
that aim at restricting the inguinal dissection area. However, all techniques present different local recurrence rates, probably due to false negative results (2).

Video-assisted surgery has been employed in the iliac and retroperitoneal lymph nodes approach, reducing postoperative discomfort, minimizing anatomic sequels and allowing a faster recuperation of patients, keeping the functional results of conventional surgery for the majority of indications.

We aimed at describing and illustrating the technical details of a minimally invasive procedure for inguinal lymphadenectomy recently described in the clinical scenario (3). This technique duplicates the principles of conventional technique, promoting a radical resection of inguinal lymph nodes, with encouraging preliminary results regarding the reduction of surgical morbidity.

\section{SURGICAL TECHNIQUE}

The technique described was developed in a prospective protocol that includes up to now 7 patients presenting penile spinocellular carcinoma, without palpable lymph nodes or that had a regression after a 6-week-antibiotic therapy. All patients had an indication of bilateral lymphadenectomy due to the presence of risk factors for lymph node dissemination such as: clinical stage $>\mathrm{T} 1$ or information regarding the initial biopsy such as histological grade $>1$, lymphatic or vascular invasion.

After signature of the informed consent the patients were submitted to classic open surgery in one of the members (control group) and a video-assisted surgery, named video endoscopic inguinal lymphadenectomy (VEIL) in the other member (group of the technique to be assessed).

\section{Control Member}

For the open conventional surgery, we have used the superficial inguinal lymphadenectomy technique and deep in the Dressler triangle, medial to the femoral artery, without the preservation of the long saphenous vein through a large inguinotomy.

\section{Video Endoscopic Inguinal Lymphadenectomy (VEIL)}

1 -Positioning and preparation of the inferior member - The leg is folded over the thigh in a way to put in evidence the femoral triangle that is marked with ink over the skin. After the marking, the leg is extended and fixed to the table with abduction and light external rotation of the thigh. The video monitor is positioned at the contralateral side to the operated one at the patient's pelvic waist.

2 - Introduction of the ports - At $2 \mathrm{~cm}$ of the femoral triangle vertex in a distal sense an incision of $1.5 \mathrm{~cm}$ in the skin and in the subcutaneous tissue until the Scarpa's fascia is performed, being developed a subcutaneous plan with scissors and later with a digital maneuver in the largest possible extension. A second incision of $1 \mathrm{~cm}$, at around $2 \mathrm{~cm}$ above and $6 \mathrm{~cm}$ medially to the first incision, to the introduction of a $10 \mathrm{~mm}$ port. It is possible to identify the trajectory of the saphenous vein through this access. A laterally symmetric position $5 \mathrm{~mm}$ port is introduced for graspers, dissection tweezers and scissors. At the initial access, a $10 \mathrm{~mm}$ Hasson trocar is preferably introduced. All the ports are fixed to the skin through a purse-string suture with cotton 0 . At the initial port, we introduce a 0 -degree optic, and at the medial port, we introduce the tweezers of the harmonic scalpel and the clipper. The surgeon and the camera operator are positioned laterally to the operated member.

3 - Expansion with gas of the working space - The creation of a working space is completed through the initial insufflation of $\mathrm{CO} 2$ with a 15$\mathrm{mmHg}$ pressure, with its fast diffusion, being able to keep the pressure at $5-10 \mathrm{mmHg}$ during the procedure (Figure-1). Transillumination allows a good orientation regarding the progression of the dissection area.

4 - Retrograde separation of the skin flap This time is fundamental to the success of the procedure and is performed with a harmonic scalpel. Initially we perform the separation between the skin and the fibroareolar tissue that contains the superficial lymph nodes until the external oblique muscle fascia on the superior part (Figure-2). Afterwards we proceed to the dissection of the fundamental parameters, having as a limit the long adductor muscle and its 


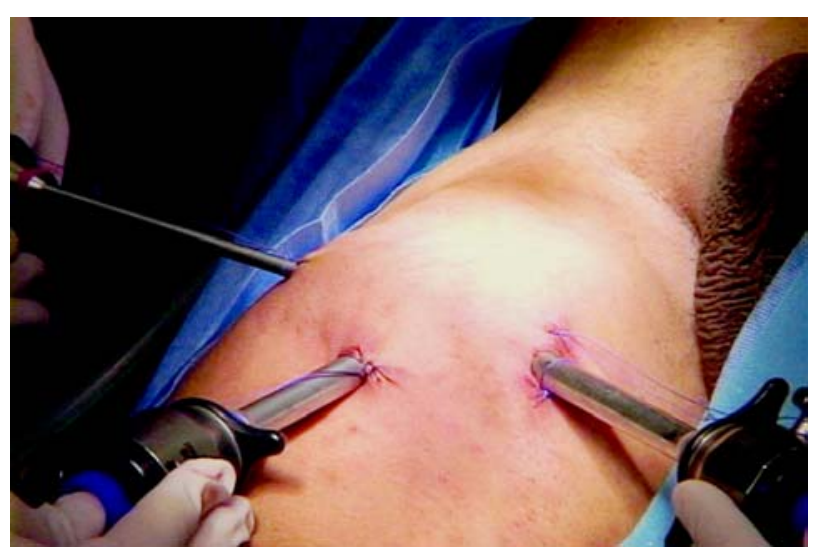

Figure 1 - Trocar disposition for a right member lymphadenectomy. The work space was almost all filled up by the diffusion of gas.

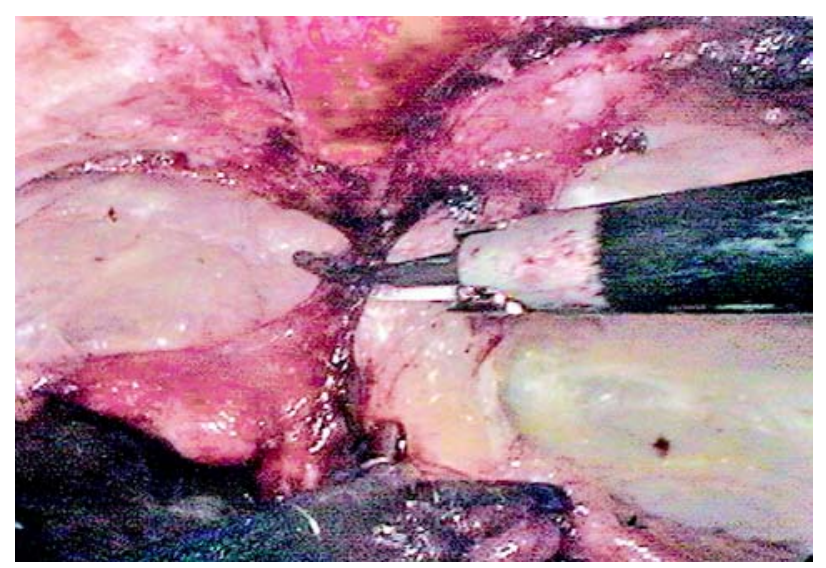

Figure 2 - Separation of the skin and the fibroareolar tissue that contains the lymph nodes, with the aid of a harmonic scalpel.

fascia medially, the sartorius muscle and its fascia laterally, and the inguinal ligament superiorly. It is possible to identify branches of the femoral nerve that should be preserved.

5 - Identification and cranial dissection of long saphenous vein until the oval fossa (Figure-3).

6 - Identification of the femoral artery - After the identification of the femoral artery and the opening of the femoral vein sheath we define the lateral limit of the dissection, allowing the access to the deep cervical lymph nodes (Figure-4). At this moment it can be necessary to control with 1 or 2 branch clips coming from the femoral artery that run anteriorly to the femoral vein.
7 - Distal ligature of the lymph node block at the femoral triangle vertex - the fibroareolar tissue is dissected with a harmonic scalpel and the control of the final section at the femoral triangle vertex is obtained with clips.

8 - Liberation of the lymph nodes until the great vessels above the femoral floor. During this operative time, the use of the harmonic scalpel and a careful manipulation of the specimen in areas near the veins are necessary to avoid vascular lesion. As in the conventional technique, the aim is to skeletonize the femoral veins, resecting all local lymphatic tissue (Figure-4).

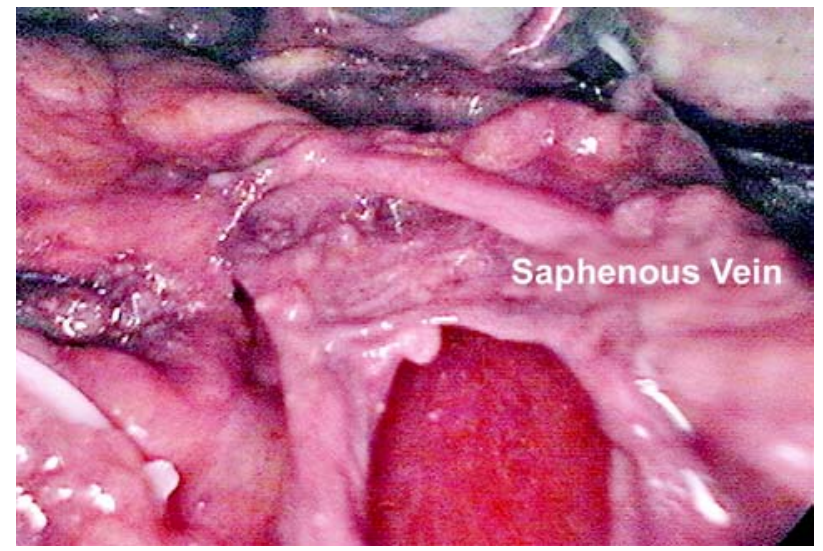

Figure 3 - Dissection of the long saphenous vein in a cranial aspect.

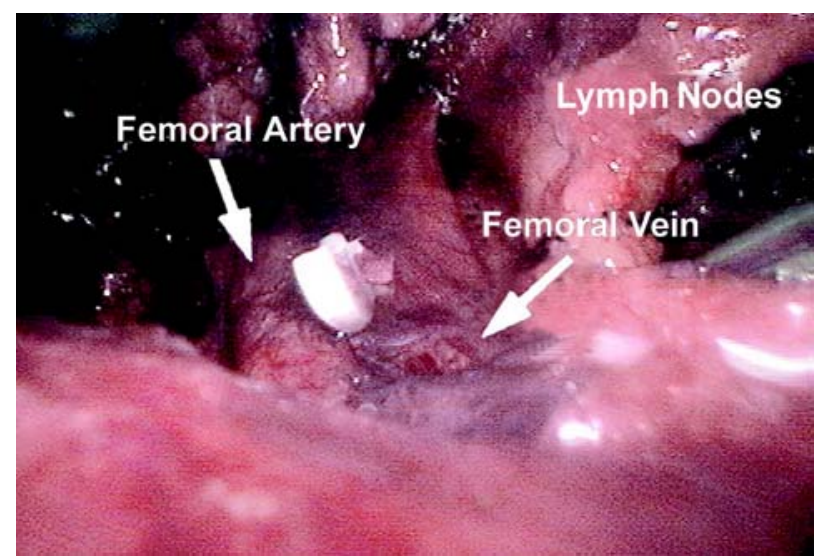

Figure 4 - Exposition of femoral artery and vein after the control of 3 small branches of the femoral artery that course anteriorly to the femoral vein. 

with clips

9 - Distal ligature of the long saphenous vein

10 - Control of the branches and the long saphenofemoral junction with a harmonic scalpel and metallic clips - most part of the branches of the long saphenous vein are controlled only by the harmonic scalpel. Branches larger than $4 \mathrm{~mm}$ need clips for the ligature. The entrance of the long saphenous vein in the femoral vein should be well dissected and controlled preferably with polymer clips (Figure-5).

11 - Final liberation of the specimen medially to the long saphenous vein, ligating the proximal portion of the lymph nodes at the deep region of the femoral channel with clips. After completing the liberation of the specimen, the endoscope view attests that all the tissue of the region was completely resected (Figure-6).

12 - Removal of the surgical specimen by the $15 \mathrm{~mm}$ incision. In case the specimen is of large dimensions, it can be put inside a bag and latter removed.

13 - Vacuum drainage through the $5 \mathrm{~mm}$ orifice and suture of the larger incisions (Figure-7).

\section{COMMENTS}

Approximately $30 \%$ of the patients with penile spinocellular carcinoma present lymph node metastasis at the time of the diagnosis. Bilateral

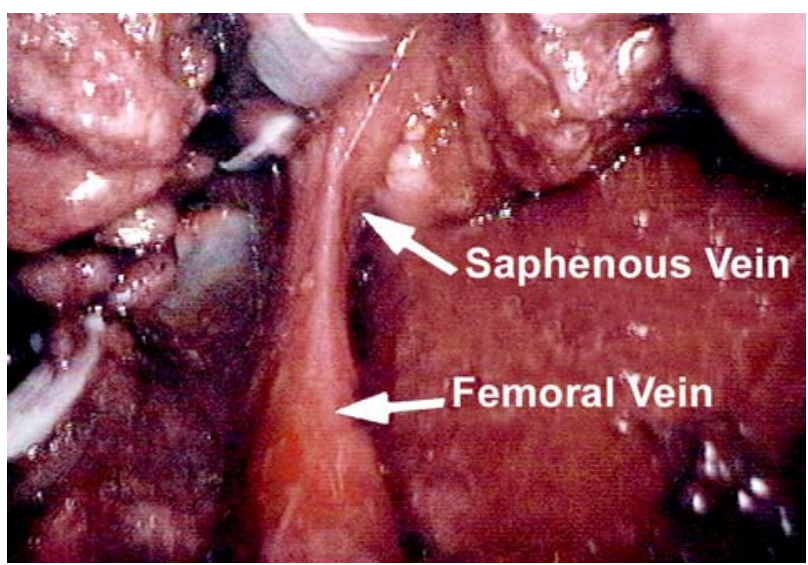

Figure 5-Aspect of the long saphenous vein in its entrance into the femoral vein.

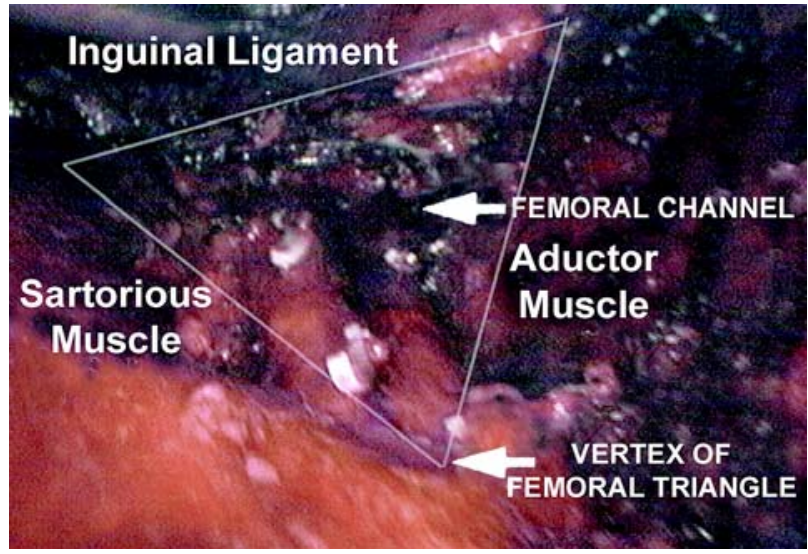

Figure 6 - Endoscopic view at the end of the lymph node dissection. The limits of the lymphadenectomy are viewed and all the lymphatic tissue was removed.

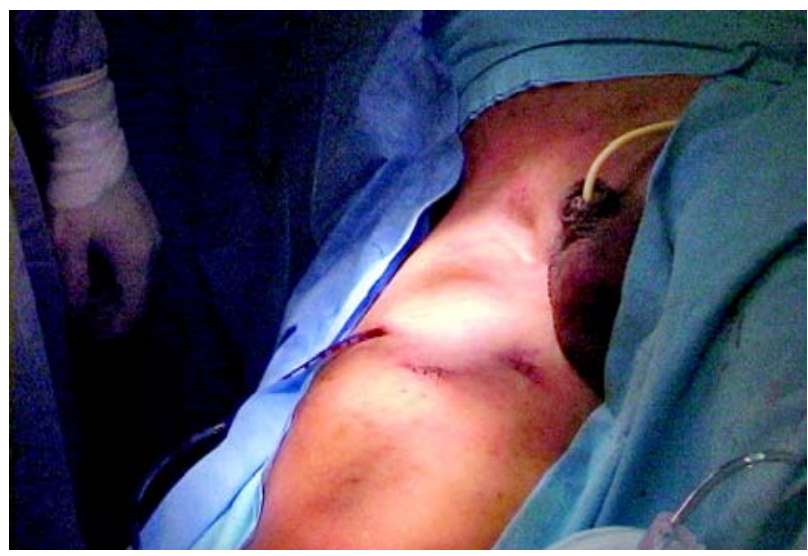

Figure 7 - Final aspect of the incisions and vacuum drainage.

inguinal lymphadenectomy is a procedure accepted as a prognostic and therapeutic value in cases of penile and urethral spinocellular carcinoma with high risk of developing metastasis (1). However, morbidity associated to this surgery is high, being questioned its need mainly when the intention is prophylactic. In the past, due to the data presented, some centers adopted a conservative conduct through a rigid clinic follow-up.

Contemporary works have demonstrated that prophylactic lymphadenectomy offers better survival 
results than salvage lymphadenectomy performed in those patients where we have initially opted for a rigorous observation. Besides that, they have also showed that the non-controlled lymph node disease was an important cause for morbidity and mortality in patients with penile cancer.

Before the dilemma of offering radical surgery with a significant morbidity to $70 \%$ of the patients in an unnecessary way or harm the survival of $30 \%$ of the patients submitted to the surveillance regimen, new alternatives were reported in literature. The techniques described in the last 20 years to reduce the morbidity are based on the reduction of inguinal dissection templates. Even though the evident reduction of operation complications described both with simplified lymphadenectomy and with the employment of sentinel lymph node with radioisotopes, some authors believe that its higher morbidity could be related to a rate of $15 \%$ of late recurrence of the disease with possible involvement of these individual's survival (2).

The present work was motivated by the attempt to reduce the complications of inguinal lymphadenectomy, based on the initial works of videoassisted saphenous vein resection, subcutaneous endoscopic procedures used in plastic surgery and video endoscopic resection of axillary lymph nodes. (4-6).

Recently, Bishoff et al. described the possibility of modified dissection of inguinal lymph nodes through endoscopic subcutaneous access performed in 2 human cadavers and in 1 patient with penile cancer stage T3N1M0. Dissection was possible on the human cadavers but it was however not possible in the patient due to the adherence of the enlarged lymph nodes to the femoral veins (7). After some technical changes we have performed, to this date, the surgery in a safe and efficient way in 7 patients with indication of prophylactic lymphadenectomy.

The idealized technique allows a complete excision of inguinal lymph nodes, the way it is done in conventional surgery, allowing an initial impression of benefit regarding the lower postoperative morbidity when compared to the conventional technique. The medium 120 minutes operative time is still superior to the open technique; however, we should consider the learning curve. Surprisingly enough, there were no skin complications. The presence of infraumbilical subcutaneous emphysema of spontaneous resolution is the rule, being uncommon the clinical manifestation. Hypercarbia can occur intraoperatively, being completely reversible with hyperventilation, without the need for conversion. In a subjective analysis, all patients preferred endoscopic surgery. Pathological exam of surgical specimens showed that the medium number of lymph nodes excised did not differ from that obtained with conventional surgery.

We attribute this preliminary result to the following technical principles: 1) Non use of electrical current and mechanical retraction with subcutaneous retractors. The retraction is performed atraumatically by the gas, minimizing cutaneous lesions, 2) Short incisions outside the area of the great vessels allow a shorter area of lesion of the separated flap and probably less chance of infection, besides making unnecessary the rotation of the sartorius muscle flap to recover femoral veins, 3) Control of the lymph nodes, visualized by magnification, with harmonic scalpel and clips. The proximal and distal ligature of major channels is fundamental to avoid important lymphoceles or lymphorea.

The presence of skin adherences or palpable mass of lymph nodes, predictive factors for technical difficulty, were excluded from this initial study which objectives were to assess the possibility and technical equivalence to classical lymph nodes resection.

\section{CONCLUSIONS}

The VEIL technique is feasible and allows the radical removal of inguinal lymph nodes at the same dissection template as conventional surgery. The main anatomic repairs of open surgery can be identified in an endoscopic view, confirming the complete removal of the lymphatic tissue within the pre-established limits.

Preliminary results with this new endoscopic approach for inguinal lymphadenectomy are promising, with potential to reduce morbidity. It seems not to change expected oncologic results with the conventional technique, but the follow-up is still short for definite conclusions. 
Future studies and validation by other authors will determine the real role of this procedure in the staging and treatment of patients with penile and urethral spinocellular carcinoma.

\section{CONFLICT OF INTEREST}

None declared.

\section{REFERENCES}

1. Bevan-Thomas R, Slaton JW, Pettaway CA: Contemporary morbidity from lymphadenectomy for penile squamous cell carcinoma: the M.D. Anderson Cancer Center Experience. J Urol. 2002; 167: 1638-42.

2. d'Ancona CA, de Lucena RG, Querne FA, Martins MH, Denardi F, Netto NR Jr: Long-term follow-up of penile carcinoma treated with penectomy and bilateral modified inguinal lymphadenectomy. J Urol. 2004; 172: 498-501; discussion 501.
3. Machado MT, Tavares A, Molina Jr WR, Zambon JP, Forsetto Jr P, Juliano RV, Wroclawski ER: Comparative study between videoendoscopic radical inguinal lymphadenectomy(VEIL) and standard open lymphadenectomy for penile cancer: preliminary surgical and oncological results. J Urol. 2005; 173: 226, Abst 834.

4. Folliguet TA, Le Bret E, Moneta A, Musumeci S, Laborde F: Endoscopic saphenous vein harvesting versus 'open' technique. A prospective study. Eur J Cardiothorac Surg. 1998; 13: 662-6.

5. Dardour JC, Ktorza T: Endoscopic deep periorbital lifting: study and results based on 50 consecutive cases. Aesthetic Plast Surg. 2000; 24: 292-8.

6. Avrahami R, Nudelman I, Watenberg S, Lando O, Hiss Y, Lelchuk S: Minimally invasive surgery for axillary dissection. Cadaveric feasibility study. Surg Endosc. 1998; 12: 466-8.

7. Bishoff JA, Lackland AF, Basler JW, Teichman JM, Thompson IM: Endoscopy subcutaneous modified inguinal lymph node dissection (ESMIL) for squamous cell carcinoma of the penis. J Urol. 2003: 169; Supl 4: 78.

Accepted after revision:

January 15, 2006

Correspondence address:

Dr. Marcos Tobias-Machado

Rua Graúna, 104/131

São Paulo, SP, 04514-000, Brazil

Fax: + 5511 3288-1003

E-mail: tobias-machado@uol.com.br 